\title{
Stabilization of the [cyclo-N5]- anion by Lewis acid-base interactions
}

\author{
Peng Wang ${ }^{1}$, Panpan $\mathrm{Wu}^{1}$, Kun Wang ${ }^{1}$, and Longjiu Cheng ${ }^{1}$ \\ ${ }^{1}$ Anhui University
}

July 23,2020

\begin{abstract}
Due to it is potential application in the field of high energy density materials, how to stabilize cyclopentazolate anion (cycloN5-) has attracted many interests theoretically and experimentally. Therefore, a series of ion salts containing [cyclo-N5]- were synthesized and studied. The instability of [cyclo-N5]- is caused by the five lone pairs of electrons localized on five neighbored $\mathrm{N}$ atoms. In this work, we expect if the [cyclo-N5]- can be stabilized by the coordination with acidic ligands, by weakening the multi repulsion from the lone pairs to stabilize the [cyclo-N5]-. The two compounds of [N5(BH3)5]-, and [N5(AgCN)5]- have been designed and compared based on the Lewis acid-base theory. [N5(H2O)5]- is designed to evaluate the effect of hydrogen bond in the stabilization. For all the structures, we study the bonding properties and thermal stabilities based on the analysis of electronic structures and Car-Parrinello molecular dynamics (CPMD) simulations. The results indicate it is a effective method to stabilize [cyclo-N5]- by introducing the Lewis acid. Our insights on [cyclo-N5]- compounds with high thermal stability under ambient conditions will provide a new idea for the research and synthesis of new high energetic [cyclo-N5]- series compounds.
\end{abstract}

\section{Hosted file}

manuscript-ijqc.doc available at https://authorea.com/users/345516/articles/471729stabilization-of-the-cyclo-n5-anion-by-lewis-acid-base-interactions 